\title{
Capsule Endoscopy Versus Colonoscopy in Patients With Previous Colorectal Surgery: A Prospective Comparative Study
}

\author{
Claudio Fiorillo ${ }^{\mathrm{a}, \mathrm{b}}$, Giuseppe Quero ${ }^{\mathrm{a}, \mathrm{b}}$, Fabio Longo ${ }^{\mathrm{a}, \mathrm{b}}$, Pietro Mascagni ${ }^{\mathrm{a}}$, \\ Michel Delvaux ${ }^{\mathrm{a}, \mathrm{c}, \mathrm{d}}$, Didier Mutter ${ }^{\mathrm{a}, \mathrm{c}, \mathrm{d}, \mathrm{e}}$
}

\begin{abstract}
Background: Colonic capsule endoscopy (CCE) derived from the video capsule endoscopy, initially proposed to explore the small bowel, has demonstrated high sensitivity and specificity for colonic polyp detection. The primary outcome of the study was to assess the safety, feasibility, and reliability of CCE after colorectal surgery. Secondary outcomes were to identify the detection rate of colonic lesions and recognition of the surgical anastomosis as compared to colonoscopy.
\end{abstract}

Methods: This is a prospective single-center study conducted over a 2 -year period. Thirty-seven patients with a history of colorectal surgery were prospectively included in this study. Each patient received both CCE and colonoscopy, performed by different operators blinded to each other's results.

Results: Thirty-two patients $(86.5 \%)$ completed the study and were included in the final analysis. All capsules were naturally expelled. In three patients $(9.4 \%)$, the anal verge was not identified during the CCE recording and the examination of the colon was considered incomplete. Surgical anastomosis was accurately identified by CCE in $78.2 \%$ of the patients versus $93.8 \%$ for colonoscopy $(\mathrm{P}=0.65)$. Thirty-eight lesions were detected in 14 patients. The sensitivity of CCE to detect colonic polyps was $95.2 \%$ (95\% confidence interval (CI): 77.3-99.2\%), the specificity $82.4 \%$ (95\% CI: 59.0-93.8\%). Positive predictive value (PPV) and negative predictive value (NPV) of CCE to detect colonic polyps were $87.0 \%$ and $93.3 \%$, respectively. No complications related to the passage of the capsule through the

Manuscript submitted July 16, 2020, accepted September 25, 2020

Published online October 31, 2020

aHU, Institute of Image-Guided Surgery, 1 Place de L'Hopital, 67091 Strasbourg, France

${ }^{b}$ Fondazione Policlinico Universitario A. Gemelli IRCCS, Digestive Surgery Unit, 8 Largo A. Gemelli, 0016, Rome, Italy

'Department of Digestive and Endocrine Surgery, University Hospital of Strasbourg, 1 Place de L'Hopital, 67091 Strasbourg, France

IIRCAD, Research Institute Against Cancer of the Digestive System, Strasbourg, France

${ }^{\mathrm{e} C o r r e s p o n d i n g ~ A u t h o r: ~ D i d i e r ~ M u t t e r, ~ D e p a r t m e n t ~ o f ~ D i g e s t i v e ~ a n d ~ E n d o-~}$ crine Surgery, University Hospital of Strasbourg, 1 Place de L'Hopital, 67091 Strasbourg, France. Email: didier.mutter@chru-strasbourg.fr

doi: https://doi.org/10.14740/gr1309 intestinal anastomosis were detected.

Conclusions: CCE proved to be safe and feasible, reporting a similar detection rate of colonic lesion compared to colonoscopy.

Keywords: Colonic capsule endoscopy; Video; Colonoscopy; Follow-up after colorectal surgery; Colorectal cancer screening; Colonic anastomosis

\section{Introduction}

The ideal screening method for colorectal lesions should be non-invasive, safe, operator-independent, well-accepted by patients, cost-effective, and highly accurate. Until now, colonoscopy remains the most effective way to detect colorectal polyps and to remove them [1].

In 2001, a video capsule endoscopy (VCE) (Given Imaging Ltd./Medtronic, Yoqneam, Israel) was proposed to investigate the small bowel in patients with obscure gastrointestinal bleeding $[1,2]$. VCE is currently indicated in the diagnostic workup of patients with obscure gastrointestinal bleeding and other intestinal conditions [3].

Colonic capsule endoscopy (CCE) is technically derived from VCE [4] and currently still under investigation for colorectal cancer screening. According to the European Society of Gastrointestinal Endoscopy (ESGE) guidelines, CCE could be used as an alternative to colonoscopy for colorectal cancer screening in average-risk patients [5]. This procedure has also been proposed as a further examination when conventional colonoscopy cannot be performed or if colonoscopy is contraindicated or refused by patients.

As technology is advancing, a second generation of CCE (CCE-2) has been released, equipped with two cameras with a wider viewing angle of $172^{\circ}$ and an adaptive frame rate ranging from four to $35 \mathrm{images} / \mathrm{s}$, depending on the speed of progression of the capsule into the colon [6]. Several studies have compared the diagnostic yield of CCE-2 and colonoscopy and concluded to a largely similar level of lesion detection [7-9].

Colonoscopy is often indicated in patients with history of colorectal surgery because of colorectal cancer screening, recurrent symptoms of abdominal pain or bowel disturbances, or 
Table 1. Bowel Preparation for CCE-2 and Colonoscopy

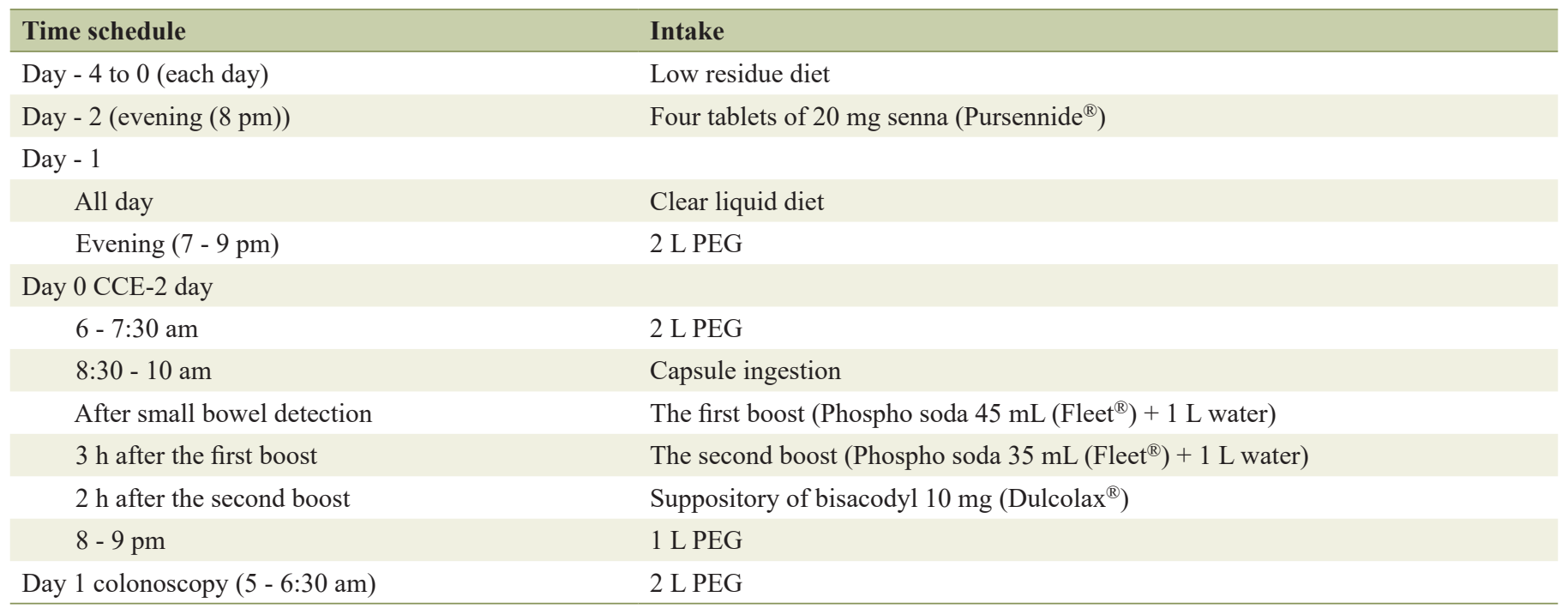

CCE-2: a second generation of colonic capsule endoscopy; PEG: percutaneous endoscopic gastrostomy.

for surveillance after resection of colorectal cancer [10]. To our knowledge, no study has evaluated CCE-2 in the followup of patients who underwent colorectal surgery. The primary aim of the present study was to evaluate the reliability and the safety of CCE-2 in these patients, the ability of the capsule to pass through the colonic anastomosis and to recognize and describe this anastomosis. The secondary aim of the study was to assess the diagnostic yield of CCE-2 for the detection of new colonic lesions. In addition, we evaluated the ability of CCE-2 to localize colorectal lesions and compared it with colonoscopy.

\section{Materials and Methods}

This is a prospective single-center study conducted over a 2-year period at the Digestive Surgery and Gastroenterology units of the Nouvel Hospital Civil (NHC) of Strasbourg. The study was approved by the National Ethics Committee (clinicaltrials.gov identifier: NCT01879943) and conducted in compliance with the ethical standards of the responsible institution on human subjects as well as the Helsinki Declaration.

\section{Selection of patients}

The inclusion criteria were as follows: patients aged $18-85$ years, with a follow-up of at least 3 months after colorectal resection with an ileocolonic, colocolonic or colorectal anastomosis, irrespective of the reason for the colonic surgery. Patients had an indication of follow-up colonoscopy, i.e., surveillance after cancer resection, recurrent abdominal pain or bowel disturbances after colonic surgery. They had no contraindication to anaesthesia and/or colonoscopy and could understand the studyrelated information for providing a written informed consent.

Exclusion criteria included the absence of a written in- formed consent, patients with a history of total colectomy, presence of terminal stoma, clinical suspicion of intestinal and/ or anastomotic stenosis (e.g., Crohn's disease), patients with a known diagnosis of Zenker's diverticulum or swallowing disorders, pregnant or breastfeeding women or women in fertile age without proven contraception, and patients with any condition precluding the reception of an informed consent.

Since no effect size is present in the literature on the reliability and safety of CCE-2 as compared to standard colonoscopy after colorectal surgery, sample size calculation was not performable.

\section{Design of the study}

The study was divided into three main phases, during which clinical and instrumental objective evaluations were performed. During the first visit to the ambulatory clinic, inclusion and exclusion criteria were checked. History and current symptoms were recorded, and the patient was examined before receiving information about the study instructions and bowel cleansing. The examinations were scheduled, and the signed informed consent was collected.

The second phase consisted of a hospital stay during which the investigations were carried out. Upon admission (day - 1), the patients started the protocol for bowel cleansing (Table 1). The next morning (day 0), the capsule was administered. On the following day (day +1$)$, all patients underwent colonoscopy under general anaesthesia with propofol (Diprivan ${ }^{\circledR}$, Astra Zeneca, Rueil-Malmaison, France) delivered by an anaesthetist, and then were discharged that same day. In this phase, any intra-procedural and early post-procedural complications were registered. Moreover, satisfaction of the patient and tolerance to CCE-2 and colonoscopy were recorded on a 10-point visual analog scale (VAS) after the procedures.

The third phase consisted of a late clinical evaluation, 15 
days to 1 month after the procedure.

\section{Bowel cleansing procedures}

The bowel cleansing procedures were administered according to the protocol validated by previous studies $[7,8]$ and detailed in Table 1. The quality of bowel cleansing was evaluated in 4 grades, i.e., excellent, good (meaning adequate cleansing), fair, or poor (the last two meaning inadequate cleansing), for both the CCE-2 recording and the colonoscopy, according to Boston Bowel Preparation Scale (BPPS) [11].

\section{Colonic capsule endoscopy}

The CCE-2 (PillCam ${ }^{\mathrm{TM}}$ Colon 2 capsule, Given Imaging Ltd./ Medtronic, Yoqneam, Israel) is made of two imagers each capturing four to 35 frames/s depending on the speed of capsule progression into the colon, a radio-frequency transmitter and a battery with a life-time of $10 \mathrm{~h}$, embedded in a $11.6 \times 32.2$ mm capsule [4].

After being fitted with eight sensor arrays on the abdominal wall, the patient was equipped with a data recorder for image storage and swallowed the capsule with a little water. After having ingested the capsule, the patient was invited to walk around in order to promote transit of the capsule. The capsule is pre-programmed in a delay mode that enables the recording of images from the oesophagus and stomach for $3 \mathrm{~min}$ after it has been swallowed. Then, the capsule automatically turns off for $1 \mathrm{~h}$ and $45 \mathrm{~min}$ in order to save power and enable a longer recording time during the transit of the capsule along the colon. When the capsule is reactivated $1 \mathrm{~h}$ and 45 min later, it is expected to be in the distal ileum.

During the whole procedure, the expulsion of the capsule was carefully monitored, and the time of expulsion was recorded.

When the recording was completed, data were transferred to a computer, where a dedicated software (Rapid software version 8, Given Imaging Ltd./Medtronic, Yoqneam, Israel) was used to obtain a video of the examination and read it. All recordings were read by an experienced gastroenterologist and reviewed by a digestive surgeon for appraisal of the surgical anastomosis. A complete examination of the colon was defined by the visualization of the anal verge during the recording.

\section{Colonoscopy}

To optimize bowel cleansing before the optical colonoscopy performed on day +1 , patients were administered $2 \times$ $1 \mathrm{~L}$ of percutaneous endoscopic gastrostomy (PEG), one in the evening after the end of the CCE-2 recording and one in the morning before the colonoscopy. Standard colonoscopies were performed by two experienced gastroenterologists having performed more than 500 procedures each and blinded to the CCE-2 results. Each colonoscopy was recorded. Any polyp detected was classified according to its location, size, and mor- phology. Polyps were removed during the colonoscopy and biopsies were obtained whenever needed. All findings were documented by pictures and video tapes.

\section{Outcomes of the study}

The primary outcome of the study was to evaluate the feasibility and safety of the use of CCE-2 in patients with previous colorectal surgery. It was defined by the number of complete examinations of the colonic mucosa by the CCE-2, down to the anal verge, the number of procedures during which the surgical anastomosis was recognized and the clinical symptoms occurring during the recording.

Secondary outcomes were to assess the rate of detection of colonic lesions by CCE-2 compared to optical colonoscopy. Reading time of CCE-2 recordings and time needed for colonoscopy (time for procedure and operating room time) were recorded.

Patient discomfort and tolerance to both CCE-2 and colonoscopy were assessed by patients on VAS $(0-10)$ on the day of the procedure.

\section{Statistical analysis}

Statistical analysis of the data was performed with Microsoft Excel 2010 (Microsoft, Seattle, WA, USA) implemented with the statistical module Analyze-it (Analyse-It Software Ltd., Leeds, UK). Respective diagnostic yields of both methods were evaluated with the Pearson's Chi-squared test. The sensitivity and specificity of CCE-2 to surgical anastomosis detection and colonic polyp detection and localisation were calculated in comparison to the optical colonoscopy which is taken as the gold standard. Ninety-five percent confidence interval (CI) was calculated for these parameters.

The positive predictive value (PPV) and the negative predictive value (NPV) of CCE-2 were calculated with respect to the prevalence of the diseases, according to Bayes' law. Agreement between CCE-2 and optical colonoscopy for diagnosing patients with at least one polyp of any size was tested with the kappa test. Concordance for the number of polyps detected by the two methods was assessed by the Spearman's test. A P value $<0.05$ was regarded as significant.

\section{Results}

\section{Study population}

Thirty-seven patients (20 men and 17 women, mean age $63 \pm$ 10 years) with a history of previous colorectal surgery were enrolled in the study. The mean time between surgery and the beginning of the study was $40.6 \pm 35.5$ months. Five patients were excluded from the final analysis as follows: colonoscopy was not carried out on two patients due to anaesthesia-related contraindications, two patients did not have a surgical anas- 
Table 2. Patient Characteristics at Inclusion

\begin{tabular}{|c|c|c|}
\hline Patient characteristics & Number (intent to treat, $\mathbf{n}=\mathbf{3 7}$ ) & Number (per protocol, $n=32$ ) \\
\hline \multicolumn{3}{|l|}{ Gender } \\
\hline Male & 20 & 17 \\
\hline Age (years, mean $\pm \mathrm{SD})$ & $63 \pm 10$ & $62 \pm 10$ \\
\hline Previous colorectal surgery & $37(100 \%)$ & $32(100 \%)$ \\
\hline \multicolumn{3}{|l|}{ Reason for exclusion } \\
\hline Contraindication to anaesthesia & 2 & \\
\hline Contraindication to colonoscopy & 1 & \\
\hline Extremely fast progression of the capsule through the colon & 1 & \\
\hline \multicolumn{3}{|l|}{ Reason for previous colorectal surgery } \\
\hline Colonic polyp & $2(5.4 \%)$ & - \\
\hline \multicolumn{3}{|l|}{ Type of anastomosis } \\
\hline Ileocolonic & $8(21.6 \%)$ & $7(21.9 \%)$ \\
\hline Colocolonic & $2(5.4 \%)$ & $1(3.1 \%)$ \\
\hline Colorectal & $26(70.3 \%)$ & $24(75.0 \%)$ \\
\hline Wedge resection & $1(2.7 \%)$ & - \\
\hline
\end{tabular}

SD: standard deviation.

tomosis and, lastly, one patient's capsule rushed down to the anus and was expelled immediately after passing through the ileocecal valve thereby precluding any examination of the colonic mucosa. Consequently, a total of 32 patients received a complete evaluation with both CCE-2 and colonoscopy, as scheduled in the protocol. Patients' clinico-demographic characteristics are reported in Table 2.

\section{Quality of bowel cleansing}

Overall, quality of bowel cleansing was considered to be adequate in 19 patients $(59.4 \%)$ on the CCE-2 recordings and in 25 patients $(78.1 \%)$ at colonoscopy (Chi-square $=1.87 ; \mathrm{P}=$ $0.17)$. There was a significant correlation between cleansing ratings for both $\mathrm{CCE}-2$ and colonoscopy $(\mathrm{kappa}=0.29 ; \mathrm{P}=$ $0.04)$.

\section{CCE-2 results}

Reading time of CCE-2 recordings was $36.2 \pm 16.2 \mathrm{~min}$. All patients ingested the capsule easily. The average time for the capsule to reach the colon, defined by the passage of the capsule through the ileocecal valve or the ileocolonic anastomosis, was $200 \pm 143 \mathrm{~min}$. A timeline of CCE-2 progression through the colon is reported in Figure 1. In three patients $(9.4 \%)$, the anal verge was not identified during the CCE-2 recording and the examination of the colon was consequently considered to be incomplete. Surgical anastomosis was accurately identified in $78.2 \%$ of the patients. In seven patients, the surgical anastomosis was not detected: six with colorectal and one with ileocolonic anastomosis.

Examples of anastomoses detected with the CCE-2 are reported in Figure 2.

\section{Colonoscopy results}

The average occupation time was $37.1 \pm 16.2$ min for colon-

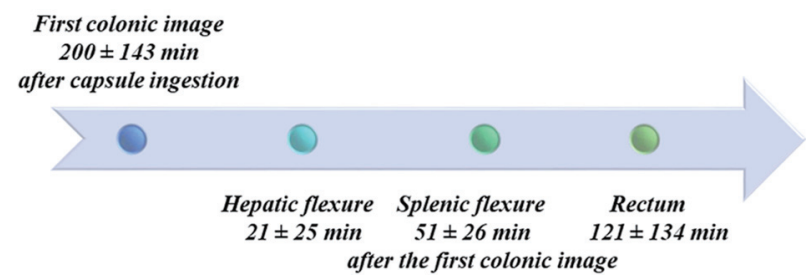

Figure 1. Colonic acquisitions timeline. Anatomical landmarks used to assess the progression of the capsule along the colon are shown with times of capsule passage. In some cases, landmarks were difficult to recognize due to alterations of the colonic lumen by the surgical resection and anastomosis, which is why these data were not analysed further. 

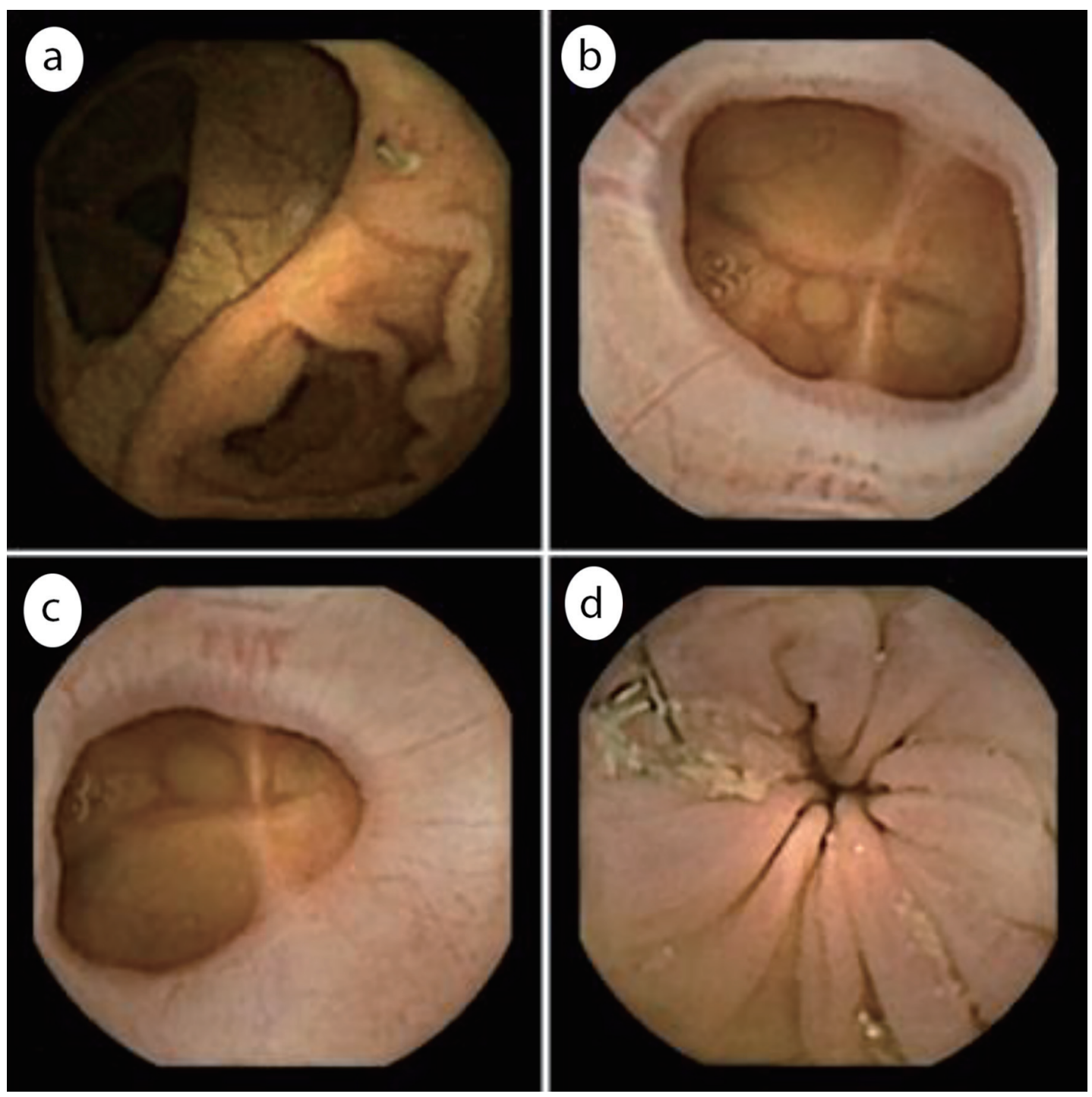

Figure 2. Examples of colonic anastomoses seen on CCE recordings. (a) lleocolonic anastomosis; (b) Colocolonic anastomosis; (c) Colorectal anastomosis; (d) Colorectal anastomosis with visible surgical staples. CCE: colonic capsule endoscopy.

oscopy in the endoscopy suite. All colonoscopies reached the cecum or the ileocolonic anastomosis. Surgical anastomosis was detected during colonoscopy in 30 out of 32 patients (93.8\%). The two anastomoses undetected during colonoscopy were both colorectal ones. There was no significant difference in diagnostic accuracy of CCE-2 and colonoscopy to detect surgical anastomosis $($ Chi-square $=0.71 ; \mathrm{P}=0.39)$.
Results of CCE-2 and colonoscopy detection accuracy are reported in Table 3.

\section{Endoscopic findings}

Thirty-eight lesions were detected in 14 patients: 18 polyps

Table 3. Detection of the Surgical Anastomosis With CCE-2 and at Colonoscopy

\begin{tabular}{lllllll}
\multirow{2}{*}{ Type of anastomosis } & \multirow{N}{*}{} & \multicolumn{2}{c}{ CCE-2 } & & \multicolumn{2}{c}{ Colonoscopy } \\
\cline { 3 - 4 } & & Accurately described & Not detected & & Accurately described & Not detected \\
\hline Ileocolonic & 8 & 7 & 1 & 8 & - \\
Colocolonic & 1 & 1 & - & 1 & - \\
Colorectal & 23 & 17 & 6 & 21 & 2 \\
\hline
\end{tabular}

CCE-2: a second generation of colonic capsule endoscopy. 
Table 4. Colonic Lesions Detected by CCE-2 and Colonoscopy

\begin{tabular}{llll} 
& CCE-2 findings & Colonoscopy findings & Total number of lesions \\
\hline Polyps & 15 & 17 & 18 \\
Diverticulosis & 16 & 16 & 18 \\
Lipoma & 2 & 2 & 2 \\
\hline
\end{tabular}

CCE-2: a second generation of colonic capsule endoscopy.

in 12 patients, two lipomas in one patient and diverticula in five patients. The respective diagnostic yield of CCE-2 and colonoscopy is shown in Table 4 . CCE-2 detected 15 polyps and colonoscopy 17 polyps. Moreover, one polyp seen on the CCE-2 recording was not found at colonoscopy. Considering colonoscopy as the gold standard, the sensitivity of CCE-2 to detect colonic polyps was $95.2 \%$ (95\% CI: 77.3-99.2\%). The specificity of CCE-2 was $82.4 \%$ (95\% CI: $59.0-93.8 \%$ ). PPV and NPV of CCE-2 to detect colonic polyps were $87.0 \%$ and $93.3 \%$, respectively.

\section{Safety and tolerance}

Four patients $(12.5 \%)$ experienced mild to moderate nausea and/or abdominal pain during the bowel cleansing protocol, all resolving spontaneously within $24 \mathrm{~h}$. Transit of the capsule into the gastrointestinal tract was uneventful in all patients.

Tolerance of CCE-2 and colonoscopy was equally good for CCE-2 (VAS score $8.50 \pm 0.71)$ and colonoscopy $(8.56 \pm$ 0.94). The comfort assessment showed a VAS score of 8.56 \pm 0.67 for CCE-2 and $8.63 \pm 0.79$ for colonoscopy, with no statistically significant difference $(\mathrm{P}=0.74)$.

\section{Discussion}

In the present study, we evaluated the feasibility, safety and tolerance of CCE-2 for examining the colonic mucosa in patients with a history of colorectal surgery resulting in a colonic anastomosis. In all patients, the capsule progressed smoothly along the gastrointestinal tract, but final analysis of the results was performed on a subset of 32 patients who completed all procedures as defined by the experimental protocol. Hindrance to the capsule during its progression along the gastrointestinal tract is one of the main concerns with the use of video capsule endoscopy for investigation of the small bowel $[2,12]$ or the colon. Obstruction to the capsule progression has been reported in up to $2 \%$ of the patients. It is more frequently observed in patients with Crohn's disease, usage of non-steroidal antiinflammatory drugs or with previous small bowel surgery. To detect the risk of capsule obstruction in the small bowel, a selfdissolving capsule, the Agile ${ }^{\mathrm{TM}}$ patency capsule (Given Imaging Ltd./Medtronic, Yoqneam, Israel), has been proposed but the predictive value of this system is not $100 \%[12,13]$. Also, imaging examinations such as computed tomography (CT) and magnetic resonance imaging (MRI) do not detect intestinal stenoses effectively [14]. In the colon, the main cause of capsule retention could be a luminal stenosis due to a tumour or a surgical anastomosis [15-17]. Until now, no case of capsule retention in the colon has been reported in previous studies of CCE $[18,19]$. Moreover, the Agile ${ }^{\mathrm{TM}}$ patency capsule is not indicated to detect colonic stenoses. Hence, a careful clinical interview remains the best method to detect patients at risk of capsule blockage.

The ability of CCE-2 to detect the colonic anastomosis and identify its type was assessed in comparison to colonoscopy. In our study, there was no statistically significant difference in the detection rate of surgical anastomoses by CCE-2 and optical colonoscopy (Table 3). However, CCE-2 detected less frequently colorectal anastomoses than colonoscopy. This may be due to a rapid progression of the capsule or a lower level of bowel cleansing in the distal colon. Handsewn, large anastomoses may also have been under-recognized by CCE-2 in the absence of colonic insufflation. By contrast, ileocolonic anastomoses were as accurately recognized by CCE-2 as by optical colonoscopy. Thorsen et al recently showed the superiority of the motility wireless capsule endoscopy in the detection of both ileocolonic junction and ileocolonic anastomoses after right hemicolectomy [20]. However, this motility capsule is used to record intestinal contractions and locates the ileocolonic junction or anastomosis based on the motility pattern, in the absence of any video-recording and therefore cannot be compared with our results.

Today, colonoscopy is considered to be the gold standard for examination of the colonic mucosa, enabling both the diagnosis and the treatment of colonic lesions, mainly colonic polyps [21]. CCE is used for detection and screening only, as treatment of any lesions found is not possible. Several studies have compared the diagnostic yield of CCE and colonoscopy in the context of colorectal cancer screening, the main measure being the rate of adenoma detection [7-9]. Sensitivity and specificity of CCE shown in these studies recently led the European Society of Gastrointestinal Endoscopy (ESGE) to propose $\mathrm{CCE}$ as a valuable alternative to colonoscopy in colorectal cancer screening [5]. In the present study, we observed sensitivity, specificity and PPV/NPV of CCE-2 for the detection of colonic polyps to be similar as those of colonoscopy, supporting the use of CCE-2 for the examination of the colon in patients with a history of colorectal surgery. According to the US National Comprehensive Network Guidelines [22], endoscopic surveillance is indicated 1 year after surgery for patients with a history of colorectal cancer or even earlier (3 months), if preoperative colonoscopy was not properly performed, in case of obstructive disease or after emergency surgery performed without any preoperative endoscopic examination [10].

However, this surveillance may be limited in some patients because of a rate of incomplete colonoscopy between 5\% and 
$24 \%$, due to inadequate bowel cleansing, difficult sigmoid loop, severe diverticular disease, or previous abdominal/pelvic surgery $[23,24]$. Koido et al have shown in their meta-analysis including 11,812 patients [25] that a history of abdominal or pelvic surgery may increase the risk of incomplete colonoscopy by $55 \%$. In our study we reported a $9.4 \%$ rate of incomplete examination of the colon by CCE-2. This finding was due to the lack of recognition of the anal verge, related to the fast passage of CCE-2 through the rectum and the anus. This result should be considered as a relative limitation of the video capsule since the distal portion of the rectum and the anus may be easily checked during physical examination. Other limitations are patient preparation which cannot be assessed before the study and probably the time of capsule ingestion which may influence its travel. Future evaluations should test this hypothesis.

We believe that CCE-2 could be a valuable complement of colonoscopy to examine the colon in its entirety especially in patients with a history of previous abdominal/pelvic surgery, allowing the detection of lesions in the colonic segments not explored by colonoscopy $[18,19]$.

Despite the small sample size of the study population, this is to our knowledge the first study evaluating the use of CCE2 in patients with a history of colorectal surgery. CCE-2 has demonstrated to be safe and feasible in this population, with a diagnostic yield comparable to that of colonoscopy. These data along with the possibility of offering a non-invasive endoscopic investigation, not requiring anaesthesia or deep sedation and colonic insufflation, suggests CCE-2 could be used as complementary method to colonoscopy, aiming at reducing the number of potentially superfluous invasive endoscopic procedures. One can imagine that controlled CCE may in the future improve the quality of this type of exploration. However, larger and comparative studies are still required to corroborate these results in order to consider CCE-2 a valuable alternative to colonoscopy.

\section{Acknowledgments}

The authors thank the nurses of the Gastroenterology Unit for monitoring the bowel cleansing procedures and for their help in the CCE-2 procedures, and the nurses of the endoscopy suite for their help during colonoscopies. The authors gratefully acknowledge Isabelle Petty for contributing to this manuscript by providing language editing and administrative assistance.

\section{Financial Disclosure}

All authors have no financial disclosure to declare in relation to this publication. Colonic capsules used in this study were provided by Medtronic as an unrestricted grant.

\section{Conflict of Interest}

All authors have no conflict of interest to declare in relation to this publication.

\section{Informed Consent}

All patients gave written informed consent to participate to the study.

\section{Author Contributions}

All authors contributed to the design and implementation of the research, to the analysis of the results and to the writing of the manuscript. The authors are accountable for all aspects of the work in ensuring that questions related to the accuracy or integrity of any part of the work are appropriately investigated and resolved.

\section{Data Availability}

The authors declare that data supporting the findings of this study are available within the article.

\section{References}

1. U.S. Preventive Services Task Force, Bibbins-Domingo K, Grossman DC, Curry SJ, Davidson KW, Epling JW, Jr., Garcia FAR, et al. Screening for colorectal cancer: US preventive services task force recommendation statement. JAMA. 2016;315(23):2564-2575.

2. Iddan G, Meron G, Glukhovsky A, Swain P. Wireless capsule endoscopy. Nature. 2000;405(6785):417.

3. Pennazio M, Spada C, Eliakim R, Keuchel M, May A, Mulder CJ, Rondonotti E, et al. Small-bowel capsule endoscopy and device-assisted enteroscopy for diagnosis and treatment of small-bowel disorders: European Society of Gastrointestinal Endoscopy (ESGE) Clinical Guideline. Endoscopy. 2015;47(4):352-376.

4. Spada C, Hassan C, Campanale M, et al. Colon capsule endoscopy. Techniques in Gastrointestinal Endoscopy 2015;17:19-23.

5. Spada C, Hassan C, Galmiche JP, Neuhaus H, Dumonceau JM, Adler S, Epstein O, et al. Colon capsule endoscopy: European Society of Gastrointestinal Endoscopy (ESGE) Guideline. Endoscopy. 2012;44(5):527-536.

6. Spada C, Hassan C, Costamagna G. Colon capsule endoscopy. Gastrointest Endosc Clin N Am. 2015;25(2):387401.

7. Spada C, Hassan C, Munoz-Navas M, Neuhaus $H$, Deviere J, Fockens P, Coron E, et al. Second-generation colon capsule endoscopy compared with colonoscopy. Gastrointest Endosc. 2011;74(3):581-589 e581.

8. Gay G, Delvaux M, Frederic M, Fassler I. Could the colonic capsule PillCam Colon be clinically useful for selecting patients who deserve a complete colonoscopy?: results of clinical comparison with colonoscopy in the perspective of colorectal cancer screening. Am J Gastroenterol. 2010;105(5):1076-1086.

9. Spada C, Pasha SF, Gross SA, Leighton JA, Schnoll- 
Sussman F, Correale L, Gonzalez Suarez B, et al. Accuracy of first- and second-generation colon capsules in endoscopic detection of colorectal polyps: a systematic review and meta-analysis. Clin Gastroenterol Hepatol. 2016;14(11):1533-1543 e1538.

10. Mulder SA, Kranse R, Damhuis RA, Ouwendijk RJ, Kuipers EJ, van Leerdam ME. The incidence and risk factors of metachronous colorectal cancer: an indication for follow-up. Dis Colon Rectum. 2012;55(5):522-531.

11. Calderwood AH, Schroy PC, 3rd, Lieberman DA, Logan JR, Zurfluh M, Jacobson BC. Boston Bowel Preparation Scale scores provide a standardized definition of adequate for describing bowel cleanliness. Gastrointest Endosc. 2014;80(2):269-276.

12. Rezapour M, Amadi C, Gerson LB. Retention associated with video capsule endoscopy: systematic review and meta-analysis. Gastrointest Endosc. 2017;85(6):1157$1168 \mathrm{e} 1152$.

13. Delvaux M, Ben Soussan E, Laurent V, Lerebours E, Gay G. Clinical evaluation of the use of the M2A patency capsule system before a capsule endoscopy procedure, in patients with known or suspected intestinal stenosis. Endoscopy. 2005;37(9):801-807.

14. Klang E, Kopylov U, Ben-Horin S, Lahat A, Yablecovitch D, Rozendorn N, Eliakim R, et al. Assessment of patency capsule retention using MR diffusion-weighted imaging. Eur Radiol. 2017;27(12):4979-4985.

15. Davis B, Rivadeneira DE. Complications of colorectal anastomoses: leaks, strictures, and bleeding. Surg Clin North Am. 2013;93(1):61-87.

16. Ambrosetti P, Francis K, De Peyer R, Frossard JL. Colorectal anastomotic stenosis after elective laparoscopic sigmoidectomy for diverticular disease: a prospective evaluation of 68 patients. Dis Colon Rectum. 2008;51(9):1345-1349.

17. MacRae HM, McLeod RS. Handsewn vs. stapled anas- tomoses in colon and rectal surgery: a meta-analysis. Dis Colon Rectum. 1998;41(2):180-189.

18. Spada C, Hassan C, Barbaro B, Iafrate F, Cesaro P, Petruzziello L, Minelli Grazioli L, et al. Colon capsule versus CT colonography in patients with incomplete colonoscopy: a prospective, comparative trial. Gut. 2015;64(2):272281.

19. Baltes P, Bota M, Albert J, Philipper M, Horster HG, Hagenmuller F, Steinbruck I, et al. PillCamColon2 after incomplete colonoscopy - A prospective multicenter study. World J Gastroenterol. 2018;24(31):3556-3566.

20. Thorsen Y, Stimec BV, Nesgaard JM, Ignjatovic D. Detecting the non-physiological, surgically tailored ileocolic anastomosis using the wireless motility capsule. A preand post-operative, prospective, within subject trial. J Neurogastroenterol Motil. 2017;23(4):585-591.

21. Dekker E, Rex DK. Advances in CRC prevention: screening and surveillance. Gastroenterology. 2018;154(7):19701984.

22. Kahi CJ, Boland CR, Dominitz JA, Giardiello FM, Johnson DA, Kaltenbach T, Lieberman D, et al. Colonoscopy surveillance after colorectal cancer resection: recommendations of the US multi-society task force on colorectal cancer. Gastroenterology. 2016;150(3):758768 e711.

23. Rathgaber SW, Wick TM. Colonoscopy completion and complication rates in a community gastroenterology practice. Gastrointest Endosc. 2006;64(4):556-562.

24. Shah HA, Paszat LF, Saskin R, Stukel TA, Rabeneck L. Factors associated with incomplete colonoscopy: a population-based study. Gastroenterology. 2007;132(7):22972303.

25. Koido S, Ohkusa T, Nakae K, Yokoyama T, Shibuya T, Sakamoto N, Uchiyama K, et al. Factors associated with incomplete colonoscopy at a Japanese academic hospital. World J Gastroenterol. 2014;20(22):6961-6967. 